\title{
A Search for Pulsating, Mass-Accreting Components in Algol-Type Eclipsing Binaries
}

\author{
D. Mkrtichian · S. -L. Kim • A. V. Kusakin • \\ E. Rovithis-Livaniou • P. Rovithis · P. Lampens · \\ P. van Cauteren - R. R. Shobbrook · E. Rodriguez • \\ A. Gamarova $\cdot$ E. C. Olson · Y. W. Kang
}

Received: 1 November 2005 / Accepted: 1 February 2006

(C) Springer Science + Business Media B.V. 2006

\begin{abstract}
We present a status report on the search for pulsations in primary componants of Algols systems (oEA stars). Analysis of 21 systems with A0-F2 spectral type primaries revealed pulsations in two systems suggesting that of the order of ten persent of Algols primaries in this range are actually pulsators.
\end{abstract}

Keywords Binaries: close $\cdot$ Binaries: eclipsing $\cdot R$ CMa TZ Eri · TZ Dra

\section{Introduction}

The pulsating components of semi-detached Algol-type systems (oEA stars) form a group of pulsators attractive for

\section{Mkrtichian $(\square)$. Y. W. Kang}

Astrophysical Research Center for the Structure and Evolution of the Cosmos (ARCSEC), Sejong University, Seoul 143-747, Korea

\section{S.-L. Kim}

Korea Astronomy and Space Science Institute, Daejeon, 305-348, Korea

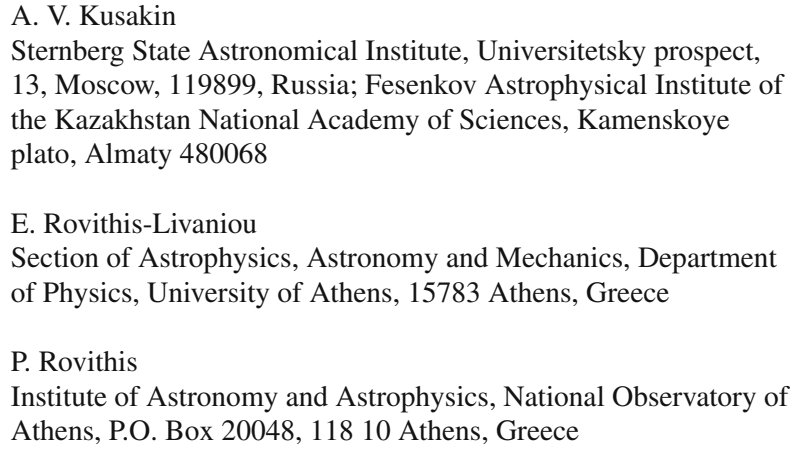

asteroseismic studies (Mkrtichian et al., 2004). The shortperiod ( $P \geq 22 \mathrm{~min}$ ) $\delta$ Scuti oscillations of A-F spectral class prime components coexist with mass accretion on their surfaces. We present here a status report on the results of an international survey to search for new pulsators in eclipsing binaries (Mkrtichian et al., 2002; Kim et al., 2003).

\section{A search for pulsations in eclipsing binaries}

The Table 1 lists the stars that have been analyzed, the spectral classes of their main components, whether the data are already published or have been collected for this program, the detection threshold and comments. In total, we analyzed

\author{
P. Lampens \\ Koninklijke Sterrenwacht van Belgie, Ringlaan 3, 1180 Brussel, \\ Belgium \\ P. Van. Cauteren \\ Beersel Hills Observatory, Laarheidestraat 166, 1650 Beersel, \\ Belgium \\ R. R. Shobbrook \\ Research School of Astronomy and Astrophysics, Australian \\ National University, Canberra, ACT, Australia \\ E. Rodriguez \\ Instituto de Astrofísica de Andalucía, CSIC, P.O. Box 3004, \\ E-18080 Granada, Spain \\ A. Gamarova \\ Thüringer Landessternwarte Tautenburg, Sternwarte 5, D-07778, \\ Tautenburg, Germany \\ E. C. Olson \\ Department of Astronomy, University of Illinois, 1002 West \\ Green Street, Urbana, IL 61801-3074, USA
}


Table 1 List of analyzed

eclipsing binary stars

\begin{tabular}{lllll}
\hline System & Sp(pri) & Publ./Obs. & $\begin{array}{l}\text { Detection threshold } \\
\text { mmag }\end{array}$ & Comment \\
\hline KO Aq1 & A0 V & P & 3.5 & No var. \\
KP Aql & A3 & P & 2.8 & No var. \\
RY Aqr & A8 & O & 5 & Uncertain \\
WW And & A5 & P & 4.4 & No var. \\
WW Aur & A3 & P & 4 & No var. \\
$R$ CMa & F0 & O & 2.7 & No var. \\
BR Cyg & A5 V & P & 2.2 & No var. \\
SW Cyg & A2 & O & 1.2 & No var. \\
RW CrB & A8-F2 V & P & 2.1 & No var. \\
V477 Cyg & A3 V & P & 3.3 & No var. \\
TZ Dra & A7 V & P/O & & var. oEA \\
TZ Eri & A5 V & P/O & & var., oEA \\
V1647 Sgr & A3 III & P & 1.28 & No var. \\
CM Lac & A2 V & P & 2.6 & No var. \\
RZ Tau & A7 V & P & 5.4 & Uncertain \\
SW Lyn & F1 V & P & 3.6 & No var. \\
TX Her & A9 V & P/O & 5 & Uncertain \\
BH Dra & A 0 & O & 9 & Uncertain \\
$\delta$ Lib & A0 IV & O & 2 & No var. \\
UX Her & A3 V & O & 6.0 & Uncertain \\
X Tri & A5-A7 V & P/O & 1.9 & No var. \\
\hline
\end{tabular}

Fig. 1 The JD 2451937 and JD $2453066 \mathrm{~V}$ filter observations of $R \mathrm{CMa}$. The data for JD 2453066 does not show the short-periodic pulsational variability

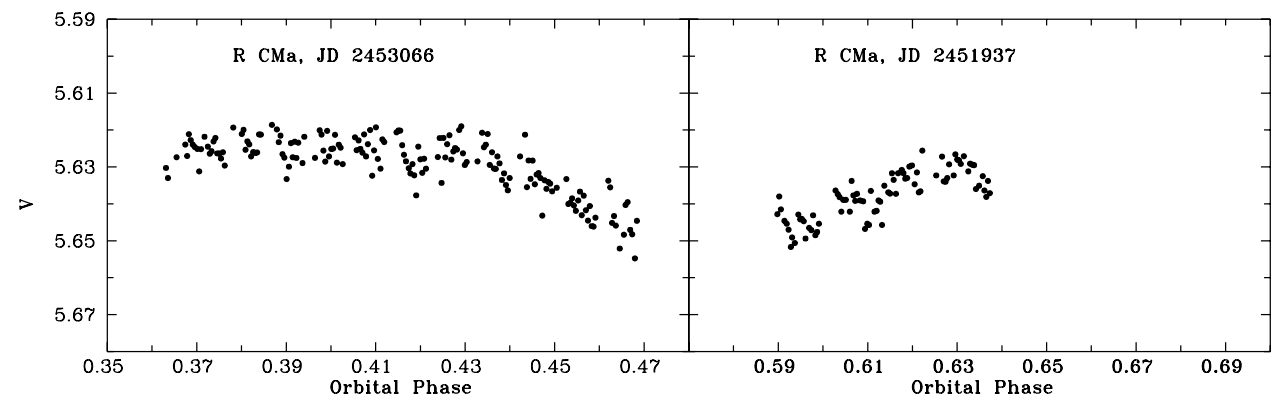

the published data for 17 eclipsing variables and obtained follow-up photometric observations for four of them. For six systems we obtained new observations. For 19 systems we obtained negative or uncertain results, two new systems TZ Eri and TZ Dra were recognized as pulsating and will be discussed together with $R$ CMa in the next subsection.

\section{1. $R \mathrm{CMa}, \mathrm{TZ}$ Eri and TZ Dra}

The orbital period of $R$ CMa system is 1.13594 days. The cooler, Roche lobe filling star has the small mass of 0.17 $M_{\odot}$, while the mass of the accreting star is $1.07 M_{\odot}$. Pulsational variability in RCMa with the period of 0.047 day $(21.21 \mathrm{c} / \mathrm{d})$ was announced by Mkrtichian and Gamarova (2000) based on the analysis of published photometric observations of Koch (1960) carried out by using the comparison star HD $56405(V=5.454, \mathrm{~A} 1 \mathrm{~V})$. To check whether the pulsational variability belongs to $R$ CMa we carried out additional observations using comparison stars HD 56316 and HD 56971, these being different from those used for Koch's observations. The data were obtained on 27 January 2001 (JD 2451937) and 1 March 2004 (JD 2453066) on the 0.48-m telescope of Tien-Shan Astronomical Observatory. The instrumental V-filter observations vs the orbital phase are shown in Fig. 1. The observations correspond to the orbital phases just before and after secondary minimum. We used for analysis the longer night on JD 2453066. As can be seen in Fig. 1, no signatures of 0.047 day period, nor any short period variability, is visible in the data. The periodogram analysis of these observations, after the removal of the trend due to the orbital variations, does not show any variability with an semi-amplitude above $2.7 \mathrm{mmag}$ in the range of frequencies $10-75 \mathrm{c} / \mathrm{d}$.

So, we cannot yet firmly disprove or confirm of existence of pulsational variability of $R$ CMa. Faced with uncertainty over the earlier results (Mkrtichian and Gamarova, 2000) we cannot now exclude the possibility that the comparison star HD 56405 ( $V=5.454$, A1V) used by Koch (1960) is 
Fig. 2 The out-of-eclipse pulsational B filter light curves of TZ Eri (left panel) and TZ Dra (right panel) phased to periods of $0.05342 \mathrm{~d}$ and $0.0204 \mathrm{~d}$ respectively
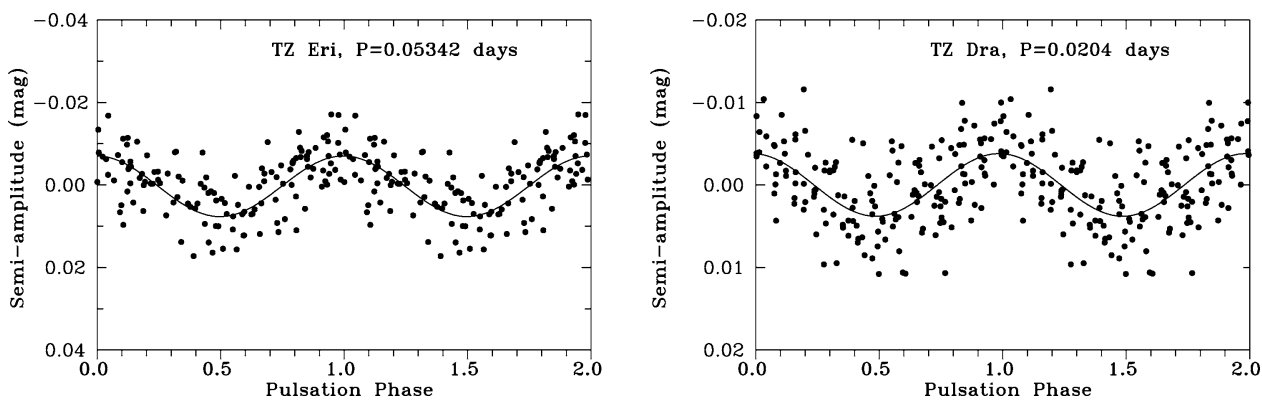

actually a low-amplitude variable star and this caused the 0.047 day variability in ( $R$ CMa-HD 56405) magnitude difference determined by Mkrtichian and Gamarova (2000). The variability of HD 56405 should be checked by further observations.

The pulsational variability of TZ Eri and TZ Dra was found by authors during the re-analysis of published photometry obtained by Burblan et al. (1998) and unpublished observations of E. Rovithis-Livaniou and P. Rovithis, respectively. The additional observations of these binaries carried out by authors in 2004 confirmed with high confidence the pulsations in these stars. In more detail, the results will be shown in further publications. Here we show (see Fig. 2) the phase curves of pulsational light variability of TZ Eri and TZ Dra folded with periods of pulsations 0.05342 day and 0.0204 day, respectively.

\section{Conclusion}

From the analysis of the out-of-eclipse light curves of 21 stars belonging to the Algol class of eclipsing binaries we have found two new pulsators: TZ Dra and TZ Eri. We do not confirm the pulsations of the primary component of $R \mathrm{CMa}$ clamed by Mkrtichian and Gamarova (2000). Based on this small sample of A0-F2 class primary components, we find that about $10 \%$ of analyzed stars are actually oEA pulsators. The results of the survey of another sample of 15 Algol-type stars carried out by Kim et al. (2005) shows that about $20 \%$ are variable. Both results show that oEA stars are promising targets for asteroseismic studies. The progress in field depends on finding and detail studying the pulsations in many Algols.

\section{References}

Barblan, F., Bartholdi, P., North, P., Burki, G., Olson, E.C.: A\&AS 132, 367 (1998)

Kim, S.-L., Lee, J.W., Kwon, S.-G., Youn, J.-H., Mkrtichian, D.E., Kim, C.: A\&A 405, 231 (2003)

Kim, S.-L., Kim, S.H., Lee, D.-J., Lee, J.A., Kang, Y.B., Koo, J.-R., Mkrtichian, D., Lee J.W.: PASPC 333, 217 (2005)

Koch, R.H.: AJ 65, 326 (1960)

Mkrtichian, D.E., Gamarova, A.Yu.: IBVS No. 4836, 1 (2000)

Mkrtichian, D.E., Kusakin, A.V., Gamarova, A., Yu., Rodriguez, E., Kim, S.L., Kim, C., Janiashvili, E.B., Kuratov, K.S., Mukhamednazarov, S.: PASPC 259, 102 (2002)

Mkrtichian, D.E., Kusakin, A.V., Rodriguez, E., Gamarova, A., Yu., Kim, C., Kim, S.-L., Lee, J.W., Youn, J.-H., Kang, Y.W., Olson, E.C., Grankin, K.: A\&A 419, 1015 (2004) 